\title{
UNIDAD DE ADQUISICIÓN DE DATOS Y MEDICIÓN BASADA EN PROTOCOLO DE COMUNICACIÓN WiFi
}

\section{DATA ACQUISITION AND METER UNIT BASED IN WiFi COMMUNICATION PROTOCOL}

\author{
Gerardo Cázarez-Ayala; Hugo Castillo-Meza y Jaime Fonseca-Beltrán \\ Profesor Investigador del Instituto Tecnológico de Los Mochis, Departamento de Ingeniería Eléctrica y \\ Electrónica, Blvd. Juan de Dios Batiz y 20 de Noviembre S/N, Los Mochis, Sinaloa.
}

\section{RESUMEN}

Sin lugar a dudas, el acelerado ritmo de competitividad de las industrias es uno de los principales factores que provocan la rápida evolución y crecimiento de las tecnologías electrónica y las comunicaciones. $\mathrm{La}$ creciente necesidad de desarrollar nuevos productos con la mejor calidad, en forma rápida y más económica, así como el asegurar e incrementar la competitividad de dichos productos en los mercados internacionales, requiere de nuevas tecnologías para la medición, monitoreo y el control de sus procesos de producción, cuya finalidad es la de mejorarlos procesos para garantizar sus productos estén presentes en el mercado en el menor tiempo posible, con la mayor calidad y bajo costo para el consumidor final.

Este trabajo se basa en el diseño e implementación de un sistema para la adquisición de datos mediante comunicación inalámbrica $\mathrm{WiFi}$, el cual está dirigido principalmente, pero no limitado a la medición, monitoreo y control de procesos de producción en la industria, aplicaciones de medición y automatización comercial y del hogar, haciendo uso de uno de los protocolos de comunicación inalámbrica con la mayor aceptación y presencia en el mercado, con lo cual, se intenta tomar Recibido: 04 de diciembre de 2011. Aceptado: 02 de marzo de 2012. Publicado como ARTÍCULO CIENTÍFICO en Ra Ximhai 8(3): $343-351$ ventaja de la infraestructura física instalada en materia de comunicaciones en el lugar de aplicación, sea casa, comercio o industria.

La Unidad está basada en el módulo de comunicaciones inalámbricas $\mathrm{RN}-131 \mathrm{G}$ de Roving Networks, el cual está habilitado para trabajar en modo local con muy bajo consumo energético dando soporte para protocolo inalámbrico WiFi con velocidades de transferencia de hasta $54 \mathrm{Mbps}$, mediante la cual es posible adquirir o medir hasta 5 variables análogas con resolución de 14 bits e incluye un sensor de temperatura y humedad relativa, medir dos señales digitales y actuar por medio de dos salidas digitales, todas ellas acondicionadas en $0-5 \mathrm{v}$ e incluye puerto USB para comunicación y/o programación.

Palabras Clave: WSN, Topología en Malla, RN131G, Inalámbrico, IEEE 802.11.

\section{SUMMARY}

Without doubt, the competitive fast rhythm of the industries is one of the main causes helped bring to the accelerated growth of the electronic technology and the communication. The need to develop new products with a best quality, cheaper and faster as assure and increase the quality of the products in the international markets, requires of new technologies to the metering, 
monitoring and control of their production processes,

of which finality is to improve the processes to guaranteed their products in the market in the less time possible, the best quality and low cost for the final consumer.

This work is based in the design and implementation of a Wireless Data Acquisition Unit based in WiFi protocol, which is oriented to meter, monitoring and the control of processes in the industry, commercial and the home automation applications, using one of the wireless protocols with the biggest acceptance in the market, with the main objective of to take advantage of the installed physical infrastructure in the place of the application, like industry, company or house.

The Unit is based in the RN-131G, a Roving Network company module, which is able to work in stand-alone mode with ultralow power consummation and supporting the IEEE $802.11 \mathrm{~b} / \mathrm{g}$ protocols, in which is possible to achieve transfer rates of up to 11 Mbps and $54 \mathrm{Mbps}$ in /b \& /g specifications respectively. Whit this unit is possible to meter up to 5 analogs signals with 14 bits precision and has include a temperature and relative humidity sensors. This unit can meter two digital signals and act mean two digitals outputs, and gas include an USB port for communication and programming tasks.

Keywords: WSN, Mesh Topology, RN-131G, Wireless, IEEE 802.11

\section{INTRODUCCIÓN}

Existen en el mercado una gran variedad de dispositivos orientados a las tareas de adquisición de datos y monitoreo de variables físicas ambientales. Estos dispositivos, son capaces de trabajar en forma distribuida, generando gran variedad de topologías de redes para comunicarse entre ellos mediante protocolos de comunicación estándares cableados como: RS-485, HART, Profibus, Ethernet and Modbus. También existen capaces de hacerlo en forma inalámbrica, resaltando protocolos de comunicación como: Bluetooth, USB Wireless, Zigbee y WiFi entre otros.

Sin duda, IEEE 802.11g ha sido la versión más aceptada por el mercado de los computadores y equipos de comunicación de uso cotidiano como PDA's y Smartphones, y ha experimentado un crecimiento dramático, ya que una de sus principales características es la de ser $100 \%$ compatible con dispositivos basados en anteriores versiones como la IEEE 802.11 b, permitiendo de esta manera la utilización de la infraestructura instalada previamente.

Este trabajo se basa en un estudio de los protocolos de comunicación inalámbricos orientados a trabajar en forma distribuida para la medición, monitoreo y control de procesos industriales y su impacto en la optimización de los procesos productivos, así como en la medición de variables ambientales. Describe de forma precisa el diseño e implementación de una unidad de adquisición de datos, con capacidad de trabajar en red mediante un enlace inalámbrico IEEE $802.11 \mathrm{~b} / \mathrm{g}$ de forma autónoma por grandes periodos de tiempo de ser necesario.

Esta unidad de adquisición de datos cuenta con diversas características, entre las cuales se destacan la capacidad de leer hasta 5 variables análogas, en un rango de voltaje de 0 a 5 volts. Con lo cual se pueden medir valores de sensores de bajo voltaje sin necesidad de requerir un acondicionamiento 
previo de la señal.

De igual forma, cuenta con la capacidad de manejar hasta 2 entradas salidas digitales acondicionadas en niveles de voltajes de 0 a 5 volts, estas pueden ser utilizadas para leer sensores digitales o modificar el estado de los actuadores utilizados en el proceso e igual número de entradas digitales.

La unidad de adquisición de datos desarrollada se basa en el módulo de comunicaciones inalámbrica $\mathrm{RN}-131 \mathrm{G}$ de Roving Networks, el cual soporta los protocolos de comunicación IEEE 802.11 $\mathrm{b} / \mathrm{g}$ y es capaz de transmitir a velocidades en rangos de 1 hasta 11 Mbps y de 6 hasta 54 Mbps en modos 802.11b y 802.11g respectivamente.

La unidad de adquisición de datos cuenta con una interfaz serial TTL de bajo voltaje para comunicar un microcontrolador de propósito general con el módulo de comunicación WiFi si se deseará aplicar control local o simplemente utilizar el módulo de comunicación inalámbrica como un simple puerto de enlace.

Este sistema fue diseñado para operar el módulo de comunicación $\mathrm{RN}-131 \mathrm{G}$ de forma autónoma principalmente, es decir, sin hacer uso de algún dispositivo inteligente como un microcontrolador, buscando con esto aprovechar todas las características que provee el módulo de comunicación inalámbrica al máximo. Para esto, es necesario llevar a cabo todas las funciones de inicialización y configuración del sistema de adquisición de datos a través de comandos remotos transmitidos a los Nodos de adquisición de datos vía radio frecuencia desde un computador a través de la red $\mathrm{WiFi}$.

Cabe destacar, que este sistema no requiere de ningún tipo de software en particular, ya que el módulo en el cual se basa cuenta con el stack de TCP/IP y ftp embebidos, facilitando con esto el desarrollo de aplicaciones inalámbricas distribuidas, siendo esto una de las principales fortalezas de este sistema de adquisición de datos.

Asimismo, el sistema desarrollado propicia el aprovechamiento de la infraestructura física e informática instalada, ya que puede utilizarse en la red existente o anexarse un nuevo segmento de red inalámbrica, permitiendo el acceso a los nodos de adquisición de datos o al equipo de cómputo o microcontrolador que actué como unidad central del sistema. También, hace posible acceder a los datos de la red con cualquier equipo de cómputo como una Laptop o Netbook, en el cual se cuente con una tarjeta de red inalámbrica o dispositivo móvil si es el caso.

Por otro lado, cabe indicar que una de las principales limitaciones del sistema es el rango de cobertura que se alcanza, el cual puede variar entre 50 y 500 metros, según se esté utilizando en interiores o exteriores, y por supuesto los obstáculos en la línea de vista. De igual manera, no es posible implementar topologías de redes avanzadas como malla o árbol sin el uso de equipos repetidores exclusivos para este fin. Siendo estas quizás sus principales limitaciones si se compara el sistema de adquisición de datos con algún otro basado en protocolo Zigbee, con el cual si es posible implementar estas topologías pero la velocidad de transferencia varia en el rango de $20 \mathrm{Kbps}$ hasta $250 \mathrm{Kbps}$.

Lo anterior creemos tendrá un gran impacto en la implementación de sistemas de control distribuido y redes de sensores inalámbricos, en el sentido que facilitará el desarrollo de estas estructuras de control y desde el 
punto de vista académico optimizará los procesos de aprendizaje, coadyuvando en el fortalecimiento del perfil de egreso de nuestros estudiantes.

Protocolo de Comunicación WiFi.

IEEE 802.11 es un estándar de comunicación inalámbrica y es orientado a la implementación de aplicaciones de adquisición de datos, medición y control de procesos, así como aplicaciones para compartir datos multimedia. WiFi como es comúnmente conocido por la mayoría de la gente, nos provee de características especiales y únicas, como son: gran ancho de banda, altas velocidades de transferencia de datos, confiabilidad y robustez de las aplicaciones desarrolladas, y por supuesto su popularidad en el mercado. Bajo este protocolo es posible alcanzar velocidades de 11 y 54 Mbps con las especificaciones /b y /g respectivamente soportadas por el módulo RN-131G.

Figura 1. Topología en Estrella para WiFi

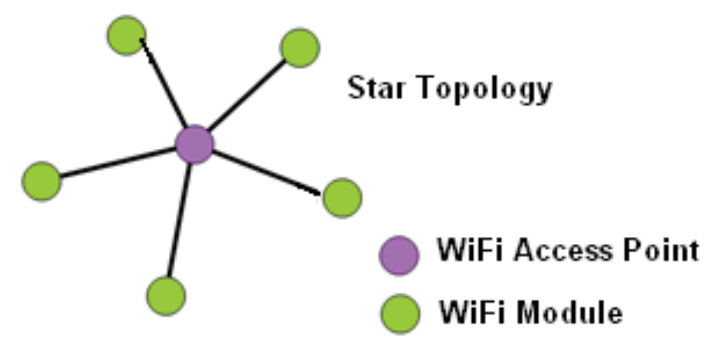

Este protocolo de comunicación maneja la capa física (PHY) y la capa de medios de accesos (MAC) del dispositivo de radio frecuencia y como en la gran mayoría de los protocolos de comunicación inalámbrica la capa de medios de acceso MAC es responsable de coordinar los accesos a uno de los canales de radio frecuencia compartidos por los dispositivos inalámbricos y módulos de comunicación en la red.
En la figura 1, se observa la topología de red en estrella, la cual puede ser implementada utilizando los nodos de adquisición de datos desarrollada. En este caso, la cobertura de los nodos es aproximadamente 400 metros en línea de vista para lugares abiertos en el exterior y 50 a 100 metros en aplicaciones para interiores, dependiendo esto de los obstáculos en el edificio, como lo son paredes, divisiones de cristal, etc.

\section{MATERIALES Y METODOS}

La tarjeta de adquisición de datos inalámbrica desarrollada está basada en el módulo RN-131G de Roving Networks, el cual, cuenta con 5 entradas analógicas y una salida para la alimentación de sensores con 3.3 volts y hasta $50 \mathrm{~mA}$, esta salida puede ser controlada por los modos de ahorro de energía del módulo. El rango de medición de las entradas analógicas es de 0 a $400 \mathrm{mV}$ soportando una entrada de voltaje de hasta 1.2 volts, utiliza una conversión análogo a digital de 14 bits con una precisión de $12 \mu \mathrm{V}$, cuando el voltaje sobrepasa los $500 \mathrm{mV}$ en las entradas analógicas $0,1,2$ y 3 el módulo despierta después de estar en modo de ahorro de energía.

Todas las entradas analógicas han sido preacondicionadas para trabajar en un rango de voltaje superior y estandarizado de $0-5$ volts. En el sistema también se encuentran otras terminales de alimentación que funcionan cuando el módulo es alimentado por USB o por medio de una fuente externa de voltaje pero no cuando se encuentra alimentado por batería.

Además se le han agregado al diseño un sensor de temperatura con un rango de medición de entre 0 y 60 grados centígrados $\mathrm{y}$ un sensor de humedad relativa del aire 
con un rango de medición de entre $0 \%$ y $95 \%$, con la finalidad de que el usuario pueda implementar de forma fácil y rápida aplicaciones que utilicen estas variables. Las salidas de voltaje de estos sensores fueron acondicionadas para bajarlas de un rango de entre 0 y 3.3 volts a un rango de entre 0 y $400 \mathrm{mV}$ para el caso del sensor de humedad; de un rango de entre 0 y 5 volts a uno de entre 0 y $400 \mathrm{mV}$ para el sensor de temperatura.

\section{Comunicación USB}

Para la comunicación cableada del módulo con una PC se utilizó un convertidor de USB a serial de la familia FTDI por medio del circuito integrado FT232RL con encapsulado superficial y un conector USB mini B. La tarjeta de adquisición de datos cuenta con la posibilidad de ser alimentada por medio de la conexión USB para lo que solo es necesario mover el selector a la posición marcada con la palabra USB.
En la figura 2, se ilustra el diagrama esquemático de la comunicación serie a través de puerto USB y su interconexión con el módulo de comunicación WiFi RN$131 \mathrm{G}$.

\section{Botoneras e indicadores visuales}

Los botones e indicadores visuales son una parte importante en el diseño del prototipo puesto que con ellos se puede visualizar el estado de funcionamiento del módulo de una forma rápida. Los principales indicadores que se le colocaron en el sistema de adquisición de datos desarrollado fueron: 1.- Indicador de asociación, para mostrar si el módulo inalámbrico se encuentra en una red; 2.- Indicador de transferencia de datos para indicar cuando el módulo transfiere información a través de él y 3.- Indicador de estado de la conexión para saber cuándo el módulo tiene correctamente configurados los parámetros de dirección IP y conexión

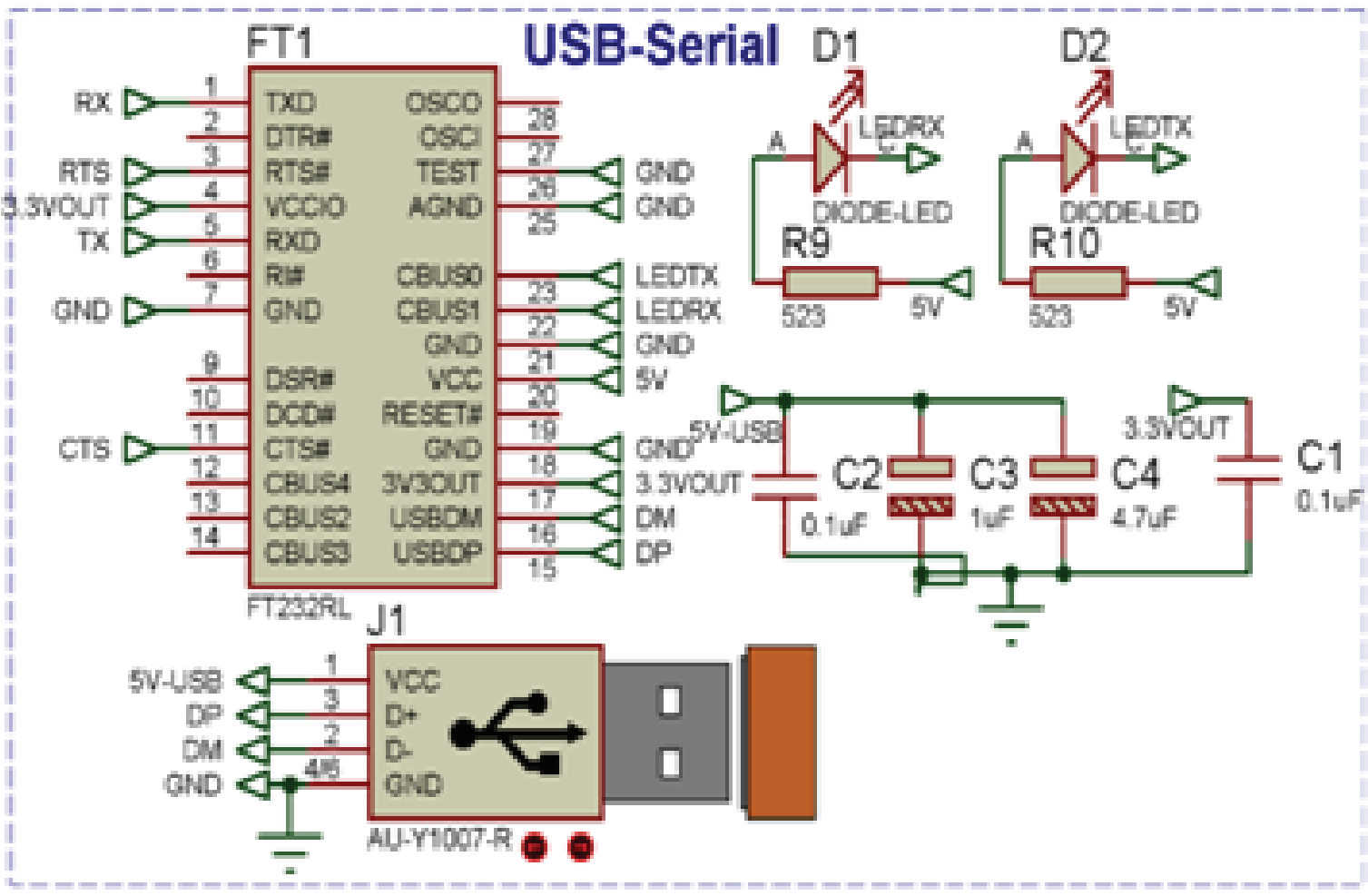

Figura 2. Esquemático puerto USB 
de datos.

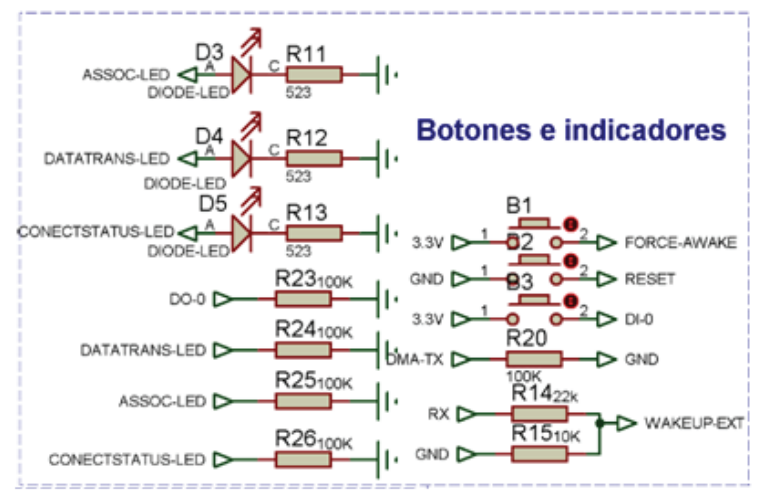

Figura 3. Esquemático de controles e Indicadores visuales.

Debido que se configuró el dispositivo para que funcionara con el mayor ahorro de energía se le agregaron resistencias de $100 \mathrm{~K} \Omega$ en configuración pull-down en cada salida digital utilizada de la forma mostrada en la siguiente imagen.

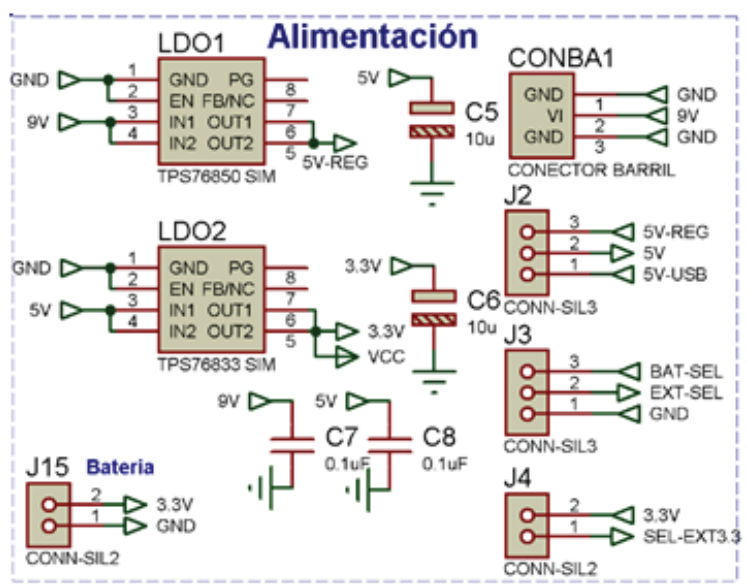

Figura 4. Diagrama de regulación de voltaje y selección de fuente.

Se agregó una medida de seguridad para cuando se encuentre el módulo en modo de ahorro de energía de forma que cuando se haga una transmisión serial el módulo despierte por medio de una entrada digital que tiene esta funcionalidad por defecto llamada WAKE UP EXT. Si el módulo no sale del modo de ahorro de energía se le agregó un segundo botón que tiene esta funcionalidad titulado FORCE AWAKE.

A su vez se le agregaron botones de RESET y un botón para forzar la entrada digital con la que cuenta la tarjeta de adquisición de datos.

\section{Alimentación}

Para la alimentación, este prototipo cuenta con distintas opciones, baterías de 9 volts, 2 baterías AA, fuente de voltaje externo como un pequeño eliminador de baterías de rango comprendido entre 6 y 10 volts o por medio del puerto USB si se encuentra conectado a algún computador. Para poder llevar a cabo esta característica se utilizaron reguladores de voltaje de alta eficiencia para 5 y 3.3 volts, con la finalidad de poder energizar dispositivos sensores y actuadores que se alimenten con estos voltajes, los cuales son los más utilizados en este tipo de aplicaciones y no limitar la funcionalidad del prototipo. La selección de la fuente de alimentación es manejada de manera física por medio de selectores instalados en la tarjeta de adquisición de datos.

\section{Adaptación de entradas analógicas}

Debido a que el módulo WiFi solo admite entradas analógicas a un voltaje máximo de 1.2 volts, estas tuvieron que ser acondicionadas para conectar dispositivos sensores con salida en voltaje en rango de 0 a $5 \mathrm{~V}$ por medio de divisores de voltaje de la manera mostrada en la figura 5. Los rangos de medición de sensores son de 0 a $400 \mathrm{mV}$ y para las entradas analógicas 0,1 , 2 y 3 al sobrepasar los $500 \mathrm{mV}$ el módulo despierta del modo de ahorro de energía. La 
resolución de las entradas analógicas es de 14 bits alcanzando una precisión de $12 \mathrm{uV}$.

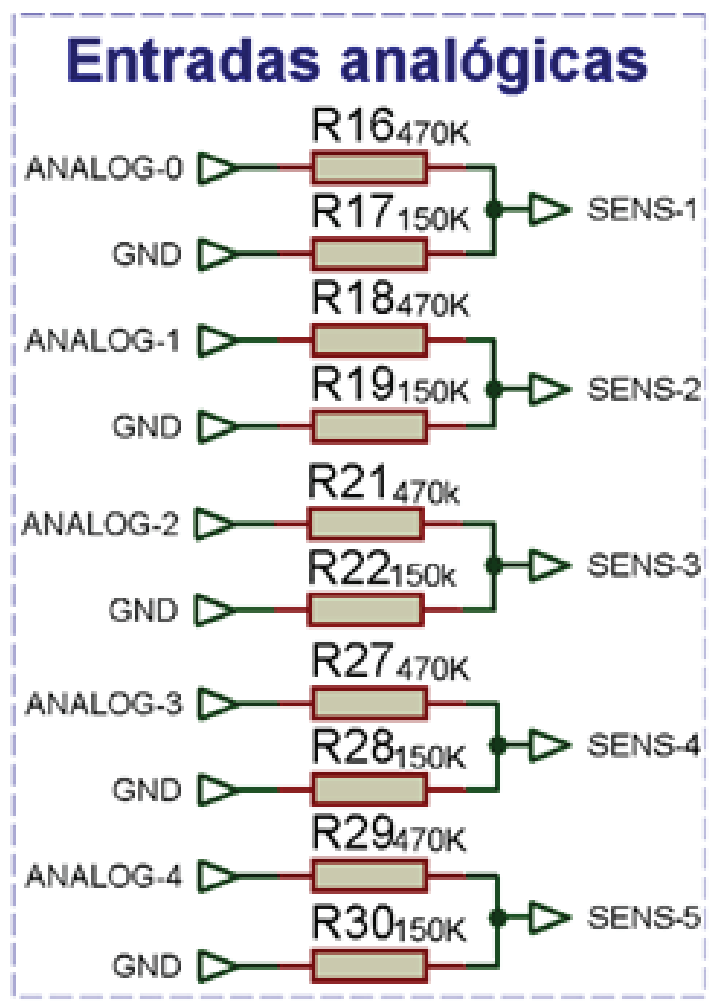

Figure 5. Acondicionamiento de entradas analógicas.

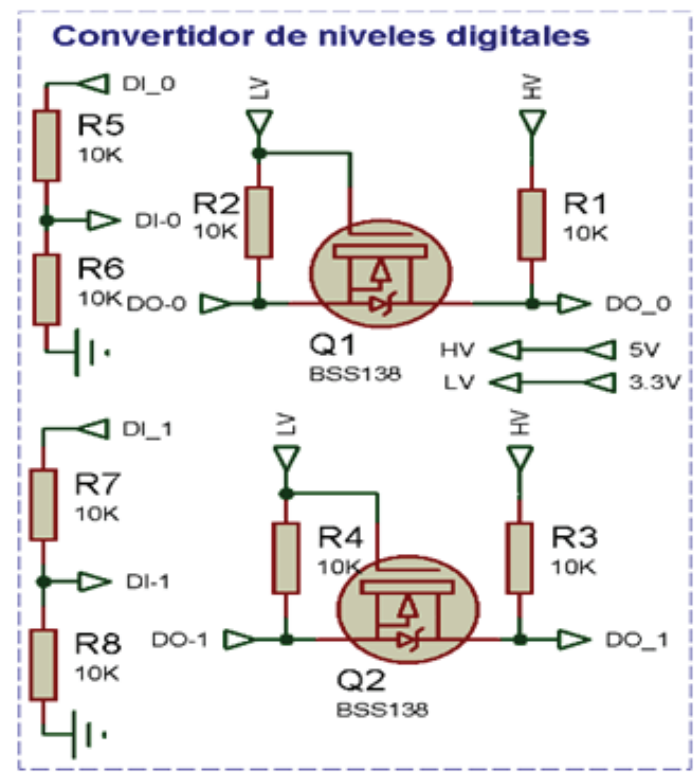

Figura 6. Acondicionamiento de entradas-salidas digitales.

\section{Niveles digitales}

Para las entradas y salidas digitales se adaptó el voltaje de operación a 5 volts por medio de un divisor de voltaje para la entrada digital y con un transistor MOSFET con número de parte BSS138 para aumentar el nivel de voltaje de 3.3 volts que es el nivel de operación del módulo WiFi a 5 volts que es el rango de operación de la mayoría de los actuadores digitales. En la figura 6 se ilustra el diagrama para el acondicionamiento de las señales de entradas-salida digitales.

\section{Sensores}

El módulo inalámbrico cuenta con dos sensores incoporados en el diseño del mismo y se encuentran conectados a las entradas analógicas 6 y 7 del módulo RN-313G correspondientes a los pines 1 y 4 respectivamente. Dado que el rango de medición aceptado por el módulo inalámbrico RN-131G es de 0 a $400 \mathrm{mV}$ se tuvo que hacer una adaptación de voltajes de operación de los sensores para que el voltaje se baje de 3.3 volts para el caso del sensor de temperatura y de 5 volts para el caso de la temperatura, para ello se utilizaron arreglos de resistencias para disminuir el voltaje.

El sensor utilizado puede realizar mediciones tanto de humedad relativa como de temperatura. Para la humedad, otorga una salida lineal de voltaje y puede medir desde 0 a $95 \%$ de humedad relativa;

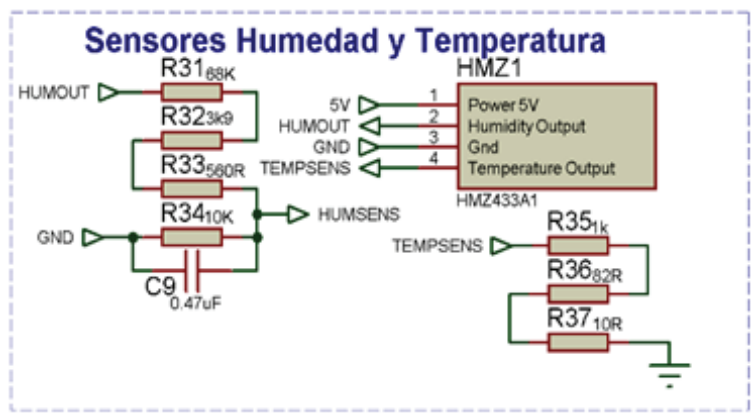

Figura 7. Acondicionamiento sensor de Temperatura y Humedad del aire. 


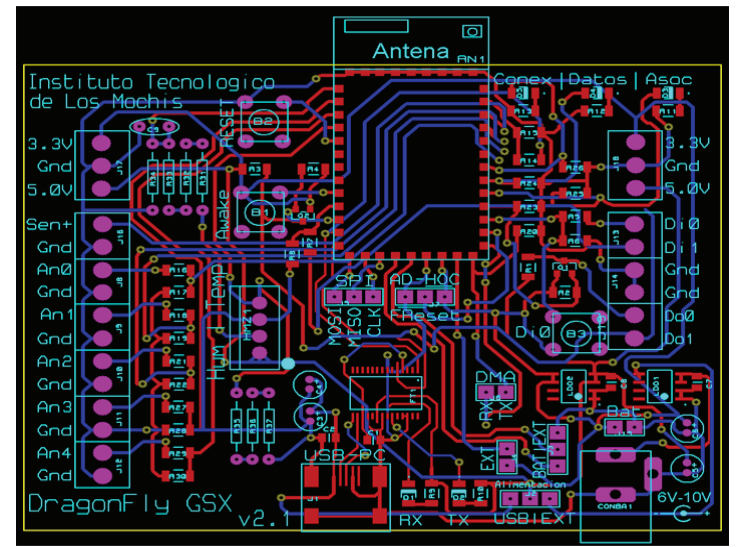

Figura 8. Diagrama de circuito impreso del prototipo desarrollado

para la temperatura este sensor utiliza un termistor, por lo que es necesario incorporar una resistencia a tierra para obtener un divisor de voltaje, el rango de medición de la temperatura va desde 0 a $60^{\circ} \mathrm{C}$. El sensor

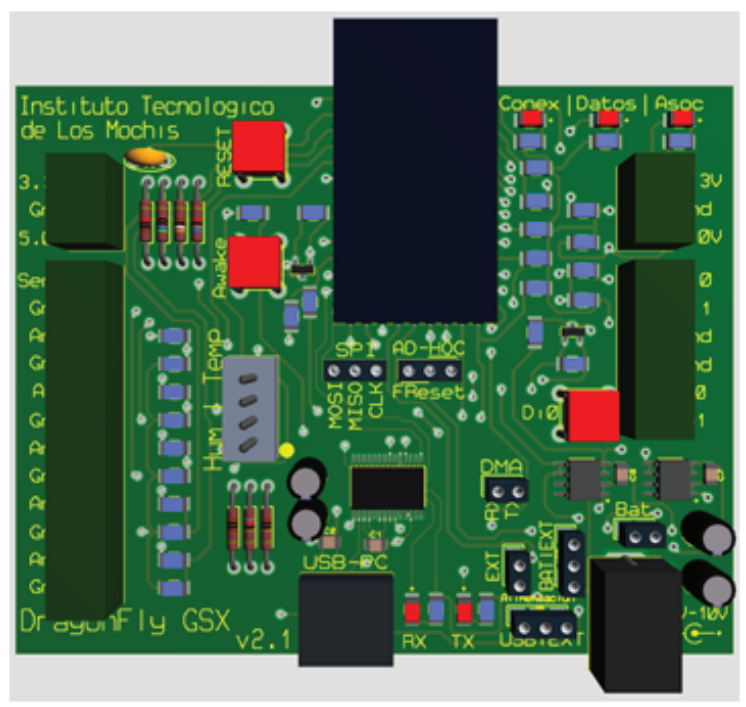

Figura 9. Diseño en 3D del Prototipo

tiene 4 terminales de las cuales dos son para la alimentación a 5 volts y las restantes se utilizan para las salidas de voltaje de los sensores de temperatura y humedad.

El diagrama esquemático de la conexión y adaptacion del sensor y los niveles de voltaje se pueden observar en la figura 7 .

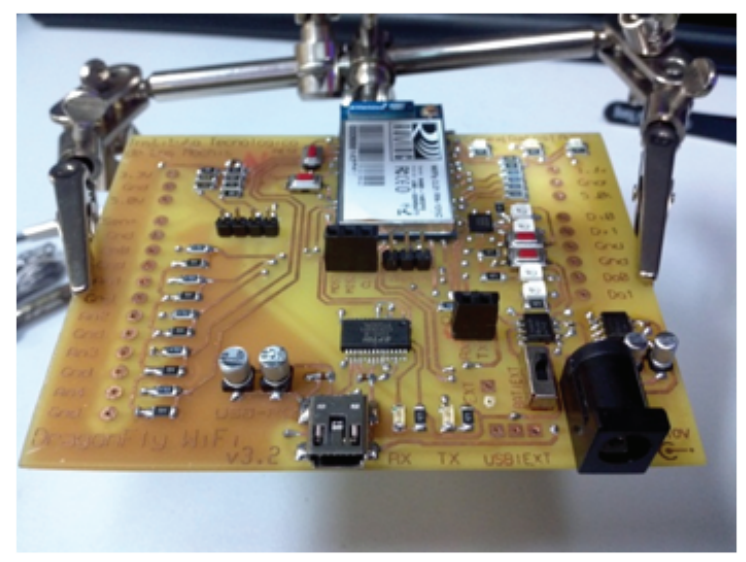

Figura 10. Prototipo en fase de desarrollo.

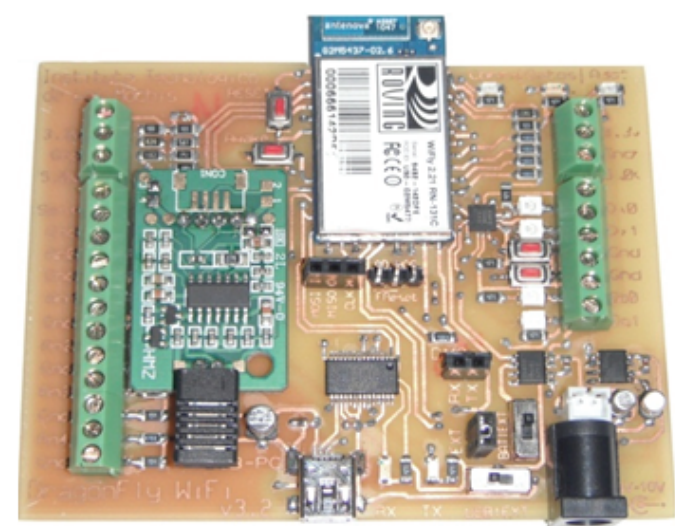

Figura 11. Prototipo WiFly terminado.

La figura 8 ilustra el diagrama PCB de la Unidad de Medición y Control de Procesos desarrollada, en la cual se pueden ver los trazos de las pistas de conexión del sistema, el cual fue implementado a doble cara y haciendo uso de circuitos integrados mediante tecnología de montaje superficial.

En lafigura 9, se muestra el diseño del sistema implementado en 3D, en este destacan los principales componentes utilizados como: el módulo de comunicación, controlador FTDI de comunicación serie USB, dispositivos de regulación de voltajes y acondicionadores de señales análogas y digitales, los bloques de terminales para conexión de sensores externos tanto análogos como digitales, asi como el punto de conexión para interface serial SPI. 
En la figura 10, se muestra el proceso de desarrollo del primer prototipo implementado. En esta figura se puede destacar el módulo de comunicación inalámbrica al fondo y el controlador FTDI para comunicación serial USB y el clásico conector USB mini B al frente.

La figura 11 ilustra el prototipo implementado completamente terminado e incluye el módulo de sensores de temperatura y humedad relativa del aire integrado en el dispositivo, el cual puede verse al centro a la izquierda en la tarjeta desarrollada.

\section{RESULTADOS Y DISCUSIONES}

Se logró diseñar e implementar un prototipo para la adquisición de datos, medición de variables físicas y supervisión de procesos de producción con capacidad de trabajar en red basado en un protocolo de comunicación inalámbrica tan común como lo es el IEEE 802.11g o WiFi.

Se realizaron diversas pruebas al prototipo, con finalidad de llevar a cabo la caracterización estática de los componentes que integra, así como la capacidad y confiabilidad de su conectividad vía inalámbrica.

Se destaca la confiabilidad al $100 \%$ en la capacidad de asociarse a una red inalámbrica previamente configurada, cuya prueba fue realizada en 1000 intentos logrando el resultado arriba mencionado.

Cabe mencionar que dichas pruebas fueron realizadas en un día normal de actividades en el laboratorio de ingeniería Electrónica del Instituto Tecnológico de Los Mochis, con otras redes inalámbricas operando en el mismo sitio. Una vez realizada la conexión a la red, inmediatamente se llevaba a cabo la adquisición de las variables de temperatura y humedad mediante los sensores embebidos en el prototipo hacia un computador vía conexión inalámbrica $\mathrm{WiFi}$, dichos datos fueron validados con éxito el $100 \%$ de los intentos realizados.

Las lecturas obtenidas de los sensores de temperatura y humedad utilizados, HMZ433A, con salida en voltaje para ambos variables, presentaron una diferencia en la exactitud de la lectura tomada en rango de: $3 \%$ a $5 \%$ para ambas variables, comparándolas contra los sensores patrones utilizados en el experimento realizado. Cabe señalar que el módulo sensor utilizado, HMZ-433A, fue seleccionado por su relación precio-precisión.

\section{CONCLUSIONES}

Este sistema fue diseñado para operar basado en el módulo de comunicación RN$131 \mathrm{G}$ de forma autónoma principalmente, es decir, sin utilizar algún dispositivo inteligente como un microcontrolador, buscando con esto aprovechar todas las características que provee el módulo de comunicación inalámbrica al máximo. Para esto, es necesario llevar a cabo todas las funciones de inicialización y configuración del sistema de adquisición de datos a través de comandos remotos transmitidos a los Nodos de adquisición de datos vía radio frecuencia desde un computador a través de la red WiFi.

Cabe destacar, que este sistema no requiere de ningún tipo de software en particular, ya que el módulo en el cual se basa cuenta con el 
stack de TCP/IP y ftp embebidos, facilitando con esto el desarrollo de aplicaciones inalámbricas distribuidas, siendo esto una de las principales fortalezas de este sistema de adquisición de datos.

De igual manera, el sistema desarrollado propicia el aprovechamiento de la infraestructura física e informática instalada, ya que puede utilizarse en la red existente o anexarse como un nuevo segmento de red inalámbrica, permitiendo el acceso a los nodos de adquisición de datos o al equipo de cómputo o microcontrolador que actué como unidad central del sistema. También, hace posible acceder a los datos de la red con cualquier equipo de cómputo como una Tablet o Laptop, en el cual se cuente con una tarjeta de red inalámbrica WiFi o cualquier dispositivo móvil, si es el caso.

Por otro lado, cabe mencionar que una de las principales limitaciones del sistema es el rango de cobertura que se alcanza, el cual puede variar entre 50 y 400 metros, según se esté utilizando en interiores o exteriores, y por supuesto los obstáculos en la línea de vista. De igual manera, no es posible implementar topologías de redes avanzadas como malla o árbol sin el uso de equipos repetidores exclusivos para este fin, siendo estas quizás sus principales limitaciones si se compara el sistema de adquisición de datos con algún otro sistema basado en protocolo Zigbee, como es el caso del sistema DragonFly diseñado por nosotros mismos, con el cual si es posible implementar estas topologías pero la velocidad de transferencia varia en el rango de $20 \mathrm{Kbps}$ hasta $250 \mathrm{Kbps}$.

Lo anterior, creemos tendrá un gran impacto en la implementación de sistemas de control distribuido y redes de sensores inalámbricos, en el sentido que facilitará el desarrollo de estas estructuras de control para aplicaciones en procesos productivos en la industria, aplicaciones en la agricultura, como medición y monitoreo de variables físicas en diversos cultivos mediante técnicas de Invernadero y aplicaciones de monitoreo de variables y automatización de tareas en el hogar o edificios inteligentes. Desde el punto de vista académico optimizará los procesos de aprendizaje, coadyuvando en el fortalecimiento del perfil de egreso de nuestros estudiantes.

\section{LITERATURA CITADA}

Yang Xiao; Yi Pan. 2009. Emerging Wireless LAN`s, Wireless PAN`s and Wireless MAN`s IEEE 802.11, IEEE 802.15, 802.16 Wireless Standard Family. John Wiley \& Sons, ISBN: 978-0471-72069-0.

Matthew S. Gast. 2005. 802.11 Wireless Networks: The Definitive Guide, Second Edition, O'Reilly Media, ISBN: 978-0596100520.

Buratti Chiara; Martalo Marco; Ferrari Gianluigi; Verdone Roberto. 2001. Sensor Network with 802.15.4 systems: Distributed processing, MAC and connectivity. Springer Berlin Heidelberg, ISBN: 9783642174896.

Holger Karl; Andreas Willin. 2005. Protocols and Architectures for Wireless Sensor Networks, John Wiley and Sons, ISBN: 978-0-470-09510-2.

\section{SITIOS DE INTERNET:}

Roving Networks, WiFly GSX 802.11b/g Datasheet, 2011, http://www. rovingnetworks.com/files/resources/ WiFly-RN-131-DS.pdf.

Roving Networks, User Manual and Command Reference for $802.11 \mathrm{~b} / \mathrm{g}$ 
wireless LAN Modules, 2011, http://

www.rovingnetworks.com/files/

resources/WiFly-RN-UM.pdf

Gerardo Cázarez Ayala, Profesor Investigador Instituto Tecnológico de Los Mochis, Departamento de Ingeniería Eléctrica y Electrónica, Blvd. Juan de Dios Batiz y 20 de Noviembre s/n, C.P. 81279, Los Mochis, Sinaloa, México.

Hugo Castillo Meza, Profesor Investigador Instituto Tecnológico de Los Mochis, Departamento de Ingeniería Eléctrica y Electrónica, Blvd. Juan de Dios Batiz y 20 de Noviembre s/n, C.P. 81279, Los Mochis, Sinaloa, México.

Jaime Fonseca Beltrán, Profesor Investigador Instituto Tecnológico de Los Mochis, Departamento de Ingeniería Eléctrica y Electrónica, Blvd. Juan de Dios Batiz y 20 de Noviembre s/n, C.P. 81279, Los Mochis, Sinaloa, México. 